\title{
Implementación de la clase invertida como propuesta metodológica en el aprendizaje de la Ley de Distribución de Planck
}

\section{Implementation of the inverted class as a methodological proposal in learning the Planck Distribution Law}

Diana Gallegos Zurita

Universidad de Guayaquil, Ecuador

Maritza Gallegos Zurita

Universidad Técnica de Babahoyo, Ecuador

Héctor Flores Nicolalde

Escuela Superior Politécnica del Litoral, Ecuador

Autor por Correspondencia: diana.gallegosz@ug.edu.ec

Fecha de recepción: 4 de Abril de 2017 - Fecha de aceptación: 5 de Junio de 2017

Resumen: En este trabajo se desarrollaron tres de las etapas de un diseño instruccional para una clase invertida, las cuales fueron: desarrollo, implementación y evaluación, como una propuesta metodológica en el aprendizaje del tópico de la Ley de Distribución de Planck. El desarrollo consistió en producir y/o seleccionar los contenidos, materiales y actividades a usar en la clase invertida; la implementación en publicar y distribuir el material desarrollado; y la evaluación (formativa y sumativa) diseñar pruebas específicas con su respectiva rubrica. En este estudio participaron cuatro estudiantes registrados en la materia de Modelos de Aprendizaje de Física, a quienes se les envió el material audiovisual con su respectiva autoevaluación desarrollado para la clase invertida, y de acuerdo a las calificaciones que obtuvieron, permitió dar una apreciación en los estudiantes de Maestría en Enseñanza de la Física (MEF) acerca de la utilidad del modelo para en lo posterior ser aplicado en sus respetivas cátedras.

Palabras clave: diseño instruccional; clase invertida; ley de distribución de planck; modelos de aprendizaje; mef

\begin{abstract}
In this work three of the stages of an instructional design for an inverted class were developed, which were: development, implementation and evaluation, as a methodological proposal in the learning of the topic of the Planck Distribution Law. The development consisted in producing and / or selecting the contents, materials and activities to be use in the inverted class; the implementation in publish and distributing the material developed; and evaluation (formative and summative), design specific tests with their respective rubric. In this study four students registered in the matter of Learning Models of Physics participated, to whom they were sent the audiovisual material with their respective self-evaluation developed for the inverted class, and according to the qualifications that they obtained, allowed to give an appreciation in the students of Masters in Teaching of Physics (MEF) about the utility of the model so that it can later be applied in their respective professorships.
\end{abstract}

Key words: instructional design; inverted class; planck's distribution law; learning models; mef 


\section{Introducción}

Con el objetivo de potenciar el aprendizaje autónomo del estudiante para que en lo posterior pueda enfrentar a situaciones reales en lo académico y profesional, así como también incentivar actitudes positivas para la adquisición de nuevos conocimientos. (López, García, Bellot, Formigós, \& Maneu, 2016)

Lo que se pretende con la clase invertida "flipped classroom" es dar una inversión al modelo tradicional donde el docente desarrolla el material en el aula, y deja las tareas y trabajos para fuera de esta, para pasar a un modelo, que con la ayuda de tecnologías de información y comunicación (TIC's), el docente desarrolle un material digital en donde el estudiante fuera del aula realice determinados procesos de aprendizaje y el profesor dentro del aula intervenga como guía para potenciar y facilitar procesos de adquisición y practica de conocimientos; de esta manera fomentar la participación activa de los estudiantes y un cambio en el paradigma del profesor.

En este trabajo se presenta tres de las etapas de un diseño instruccional para una clase invertida, las cuales son: desarrollo, implementación y evaluación, como una propuesta metodológica en el aprendizaje de la Ley de Distribución de Planck. El desarrollo consiste en producir y/o seleccionar los contenidos, materiales y actividades a usar en la clase invertida; la implementación en publicar y distribuir el material desarrollado; y la evaluación (formativa y sumativa) diseñar pruebas específicas para analizar los resultados de la clase invertida.

Cabe mencionar que todas estas etapas se realizaron fuera del aula en donde el docente fue el encargado de la creación, distribución y evaluación de dicho material digital educativo. El tópico escogido fue la Ley de Distribución de Plank, de la materia de Termodinámica Estadística, un caso de estudio con profesores de Maestría de Enseñanza de la Física.

Las siguientes etapas del esquema de clase invertida se dan dentro del aula en donde el docente crea el escenario en el cual los estudiantes despejan dudas y se profundizan contenidos en un aprendizaje activo y colaborativo a través de actividades y/o estrategias/modelos de aprendizaje, y se conviertan en protagonistas de su propio aprendizaje.

\section{Antecedentes y beneficios de la clase invertida.}

Bergmann y Sams (2012) profesores de Química en Colorado (EE.UU) introdujeron en las aulas en el año 2007 y delinearon los elementos del modelo de clase invertida: un modelo que permite una mayor interacción entre estudiantes y profesores dentro de la clase, fomentando así el aprendizaje autónomo de los alumnos fuera de esta. (Bergmann \& Sams, 2012)

Eric Mazur, docente de la Universidad de Harvard junto con Catherine H. Crouch, en el año 1991 desarrollaron una metodología llamada Peer Instruccion (Instrucción en Pares): un esquema en donde el estudiante trabaja con un material de estudio antes de la clase presencial y en el salón de clase realiza pequeñas lecciones y responde preguntas conceptuales. (Crouch \& Mazur, 2001). Mazur es uno de los pioneros del Aula Invertida, desde el año 2012 implementa Active Learning (Aprendizaje Activo), y en el año 2002, el Instituto Tecnológico de 
Massachusetts (MIT), aplica una metodología similar denominada TEAL - Technology Active Learning (Aprendizaje Activo con Apoyo Tecnológico), lo que se busca con estas metodologías es incentivar una mayor participación de los estudiantes fuera y dentro de la clase, además de fortalecer el trabajo en grupo.

En Ecuador en el año 2016 la Escuela Superior Politécnica Litoral introduce en las clases de Física $\mathrm{C}$ un modelo fundamentado en estas últimas metodologías, llamado PPL - Peer Project Learning (Aprendizaje en Pares y Proyecto; este modelo fue desarrollado por Forencio Pinela y Young-jin Seo, y supervisada por E. Mazur en el periodo (2014-2015) en la Universidad de Harvard: esta enseñanza se basa en el desarrollo de proyectos y el uso de tecnologías de información y comunicación, que permite la interacción de estudiantes y profesores dentro y fuera del aula de clases. (Leon, 2016)

En la actualidad, existen resultados de investigaciones cuantitativas de la implementación de todos estos modelos o metodologías en diferentes niveles de educación, en las cuales se evidencian un mejor desempeño académico en los estudiantes. Tal es el caso de la investigación hecha en una clase masiva de Física de la Universidad de British Columbia, DesLauriers, Schelew y Wieman (2011), compararon dos grupos de estudiantes, un grupo experimental que recibió clases invertidas con otro de control que recibió clases tradicionales. Como resultado del experimento, el grupo experimental evidenció considerables aumento en el desempeño.

\section{Materiales y Método}

\section{Participantes}

Los sujetos a quienes se envió el material audiovisual y la respectiva evaluación fueron cuatro estudiantes registrados en la materia de Modelos de Aprendizaje de Física de la III promoción de la Maestría de Enseñanza de la Física de una universidad ecuatoriana. Los estudiantes fueron dos hombres y dos mujeres que trabajan como docentes en nivel medio y superior, cuyas edades estaban comprendidas entre los 30 y 45 años y reciben las clases de maestría los fines de semana.

\section{Tareas y Materiales}

La asignatura seleccionada fue Termodinámica Estadística y tarea instruccional para este estudio fue la unidad de la Ley de distribución de Planck. Todo el material desarrollado fue enviado vía web. Para la revisión del video y la solución de la prueba se dedicó un tiempo de una semana de sábado a sábado.

\section{Procedimiento}

Luego de seleccionar la tarea instruccional, se desarrollaron las siguientes actividades:

$\checkmark$ Producción de un material audiovisual que permita al alumno estudiar preliminarmente y de forma autónoma. 
$\checkmark$ Diseño y elaboración de las preguntas de autoevaluación que deberán completar los estudiantes tras la visualización del vídeo, para diagnosticar la comprensión de dicho material y la detección de vacíos en el proceso de aprendizaje.

$\checkmark$ Distribución de los materiales digitales.

$\checkmark$ Diseño de la rúbrica de evaluación.

Para producir el video se utilizó el software libre Camtasia Studio 8 y para la elaboración del cuestionario Microsoft Word; la distribución del material a los estudiantes se hizo mediante correo electrónico y la plataforma institucional. La resolución de estas preguntas fueron enviadas por cada uno de los estudiantes a través de los mismos medios al profesor, para el cual tuvieron un tiempo de una semana. Luego se calificó y se envió la solución junto con la rúbrica para su revisión. Anexo No. 1

\section{Resultados y Discusión}

Para realizar el video primero se elaboró las diapositivas que conformaría la parte visual del mismo, luego se hizo varias pruebas de grabación para su edición final; este material tuvo una duración de aproximadamente diez minutos, en la figura 1 se muestran las capturas de pantallas de algunos momentos del video de la explicación del tópico de la Ley de Distribución de Planck. El video tenía que ser visualizado por los estudiantes antes de asistir a clases y haber contestado el cuestionario de autoevaluación.

Utilizando esta metodología las calificaciones que obtuvieron basadas con los criterios de evaluación de la rúbrica se detallan en la tabla No.1, en donde se puede ver que la calificación media fue de 4.4 sobre 10 puntos.

Los resultados obtenidos en la autoevaluación sirvieron para detectar los vacíos y falencias del material elaborado en el proceso de aprendizaje y dar una apreciación a los docentes evaluados sobre la utilidad de la clase invertida en la enseñanza de la cátedra de Física.
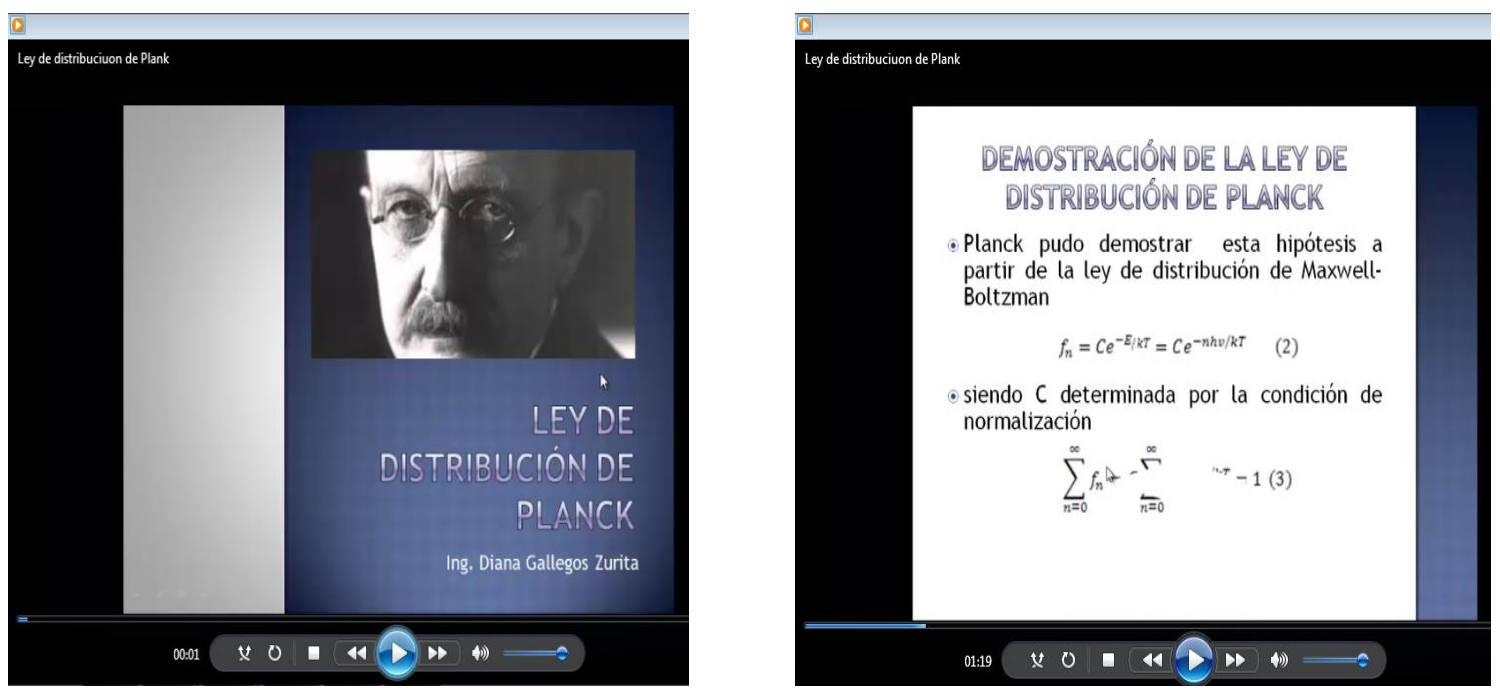

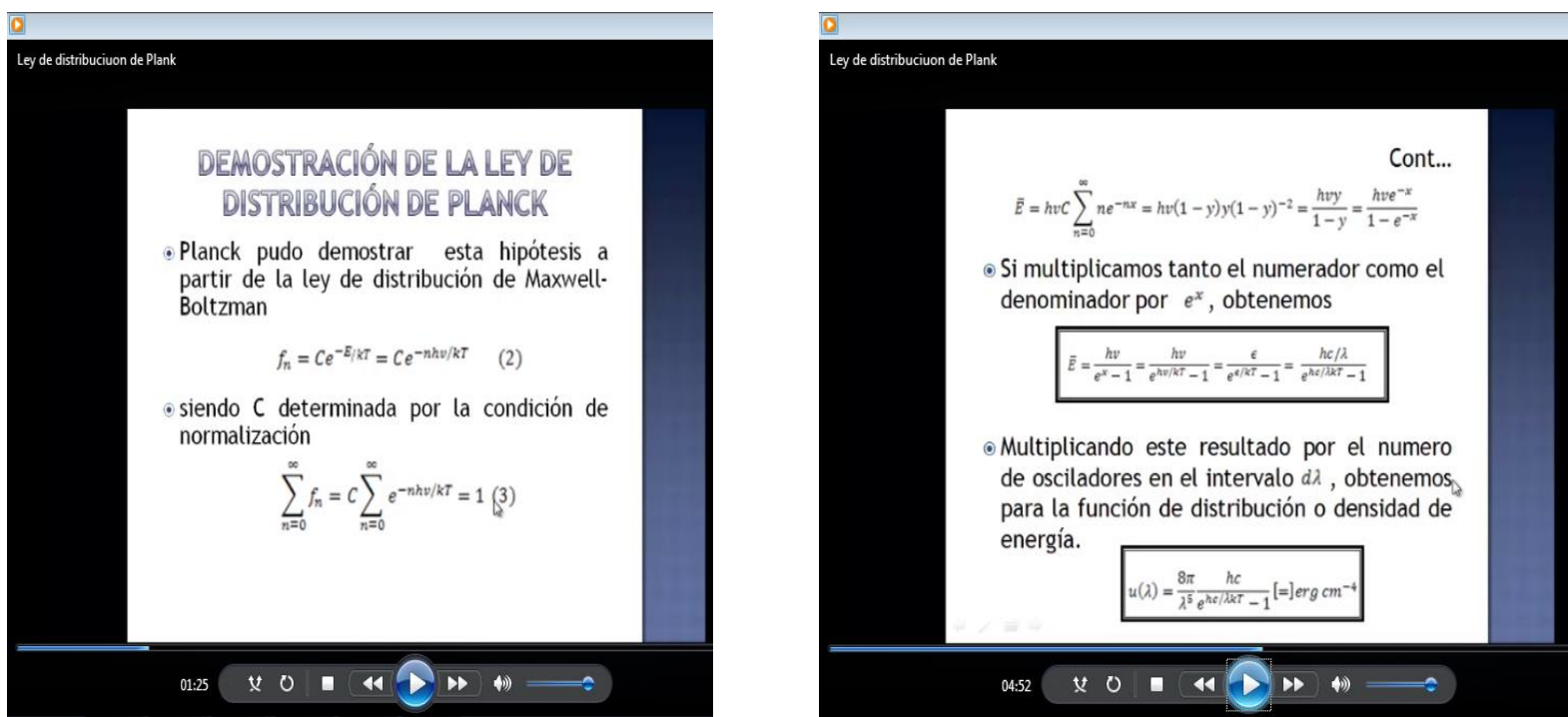

Figura 1. Capturas de pantalla del video elaborado para la clase invertida de la Ley de Distribución de Planck.

Tabla 1. Calificaciones obtenidas en el cuestionario de autoevaluación de la clase invertida.

\begin{tabular}{|c|c|c|c|c|c|}
\hline \multirow[t]{2}{*}{ Alumnos } & \multicolumn{4}{|l|}{ Temas } & \multirow[t]{2}{*}{ Total } \\
\hline & 12 & 3 & 4 & 5 & \\
\hline Marco N. & 1 & 3 & 4 & 1 & 10 \\
\hline Silvia C. & 1 & 1 & 1 & 1 & 5 \\
\hline Rosa C. & 1 & 1 & 1 & 0 & 4 \\
\hline Arsecio B. & 11 & 1 & 0 & 0 & 3 \\
\hline \multicolumn{5}{|c|}{ Media } & 4.4 \\
\hline
\end{tabular}

\section{Conclusiones}

La clase invertida tienen como objetivo potenciar el aprendizaje autónomo del estudiante e incentivar actitudes positivas para la adquisición de nuevos conocimientos con el fin de mejorar los resultados de aprendizaje, sin embargo las calificaciones que se obtuvieron en la evaluación sumativa no reflejan un buen desempeño en tres de los cuatro estudiantes evaluados.

El objetivo de desarrollar las actividades de las tres etapas del diseño instruccional para una clase invertida e implementar fue presentar una propuesta metodológica diferente en el aprendizaje de la Ley de Distribución de Planck, lo cual permitió dar una apreciación en los estudiantes de MEF acerca de la utilidad del modelo para en lo posterior poder utilizar la metodología de clase inversa en diversas cátedras y mejorar así los resultados alcanzados en una clase tradicional.

En efecto, este trabajo es un referente para mejorar los procesos de aprendizaje y proporciona de herramientas necesarias para estructurar una clase invertida dentro del aula. Las actividades y/o estrategias de aprendizaje que se escojan para estructurar la clase dentro del salón deben permitir despejar dudas y profundizar contenidos en un aprendizaje activo y colaborativo. El desarrollo y aplicación de estas actividades se presentaran en un próximo trabajo para de esta manera completar cualquier diseño instruccional que se elija. 


\section{Bibliografía}

Alkins, \& Paula, d. (2006). Quimica Fisica (8ava. Edicion ed.). Madrid: panamericana.

Bergmann, J., \& Sams, A. (2012). Flip your Classroom. United States of America: International Society for Technology in Education.

Crouch, C. H., \& Mazur, E. (2001). Peer Instruction: Ten years of experience and results. American Association of Physics Teachers, 69(9), 970-977.

Deslauriers, L., Schelew, E., \& Wiemen, C. (2011). Improved Learning in a Large-Enrollment. Science, 1-29.

Jesus Biel, G. (1998). Formalismos y metodos de la Termodinamica. Barcelona: Reverté.

Kittel. (1973). Fisica Termica. Barcelona: reveré.

Leon, V. (2016). PPL, nueva forma de enseñar y apreder la ciencia. Focus Espol, 36-37.

Llorens, J., \& FJ, S. (1997). Una Interpretacion de las Dificultades en el Aprendizaje del Concepto Integral. Divulgaciones Matematicas, 5(1/2), 61-76.

López, D., García, C., Bellot, J., Formigós, J., \& Maneu, V. (2016). Elaboración de material para la realización de experiencias de clase inversa (flipped classroom). Innovaciones metodológicas en docencia universitaria: resultados de investigación (págs. 973-984). España: Universidad de Alicante.

NORMAND, \& LAURENDEAU. (2005). Statistical Thermodynamics. New York: Cambridge University Press.

Tipler, P. (2003). Fisica Moderna. España: Reverté.

\section{ANEXO No.1}

\section{Respuestas y rubrica de la Autoevaluación de la Ley de Distribución de Planck.}

1. La energia minima que se debe suministrar a los osciladores para exitarse, debe ser de al menos un hv. (1 Punto)

a) Falso

b) Verdadero

2. La hipótesis de cuantización de Planck anula la contribución de los osciladores de alta frecuencia (longitud de onda corta), dado que estos no pueden ser excitados de forma significativa con la energía disponible. (1 Punto) 

a) Falso
b) Verdadero

3. ¿Cuál es la energia $\bar{E}$ para un oscilador que tiene una frecuencia $f=k T / h$ de acuerdo con el calculo de planck?

\section{Rubrica}

\section{Tema}

Calcula la Energía media a una frecuencia $f=k T / h$

$$
\begin{gathered}
v=f=k T / h \\
\epsilon=h f=k T \\
\overline{\boldsymbol{E}}=\frac{h v}{e^{h v / k T}-1}=\frac{\epsilon}{e^{\epsilon / k T}-1}=\frac{k T}{e^{1}-1}=\mathbf{0 . 5 8 2} \boldsymbol{k T}
\end{gathered}
$$

\section{Criterios}

Escribe la ecuación de la energía media en función de la frecuencia $\bar{E}=\frac{h v}{e^{h v / k T}-1} \quad(1.5 \mathrm{pt})$ Calcula el valor de $\bar{E}(1.5 \mathrm{pt})$

Escribe la ecuación de la energía media en función de $\lambda$. $\bar{E}=\frac{h c / \lambda}{e^{h c / \lambda k T}-1} \quad(1 \mathrm{pt})$
Puntaje (3 Puntos)

3

1

4. Grafique en un diagrama de Distribución de Energía vs Longitud de Onda para un cuerpo cuya temperatura es de $1600 \mathrm{k}$

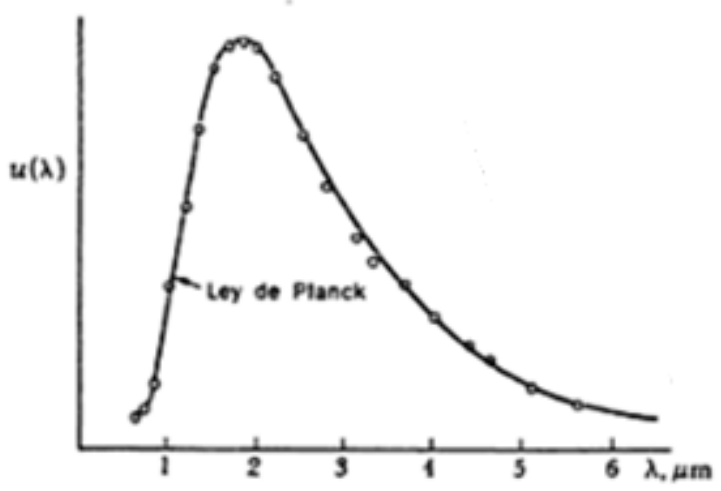

$u(\lambda)=\frac{8 \pi}{\lambda^{5}} \frac{h c}{e^{h c / \lambda k T-1}}[k J / n m]$

\section{Rubrica}

\section{Tema}

Criterios

Puntaje (4 Puntos) 


\begin{tabular}{|c|c|c|}
\hline \multirow[t]{3}{*}{$\begin{array}{l}\text { Realiza el grafico de la Distribución de } \\
\text { Energía vs Longitud de Onda para un } \\
\text { cuerpo cuya temperatura es de } 1600 \text { k }\end{array}$} & $\begin{array}{l}\text { Escribe la ecuación que define la gráfica de la } \\
\text { distribución de energía (1pt) } \\
\qquad u(\lambda)=\frac{8 \pi}{\lambda^{5}} \frac{h c}{e^{h c / \lambda k T}-1}[\mathrm{~kJ} / \mathrm{nm}] \\
\text { Realiza el grafico de la Distribución de Energía vs } \\
\text { Longitud de Onda para un cuerpo cuya temperatura } \\
\text { es de } 1600 \mathrm{k} \text { (2pt) } \\
\text { Ilustra a que longitud de onda se obtiene la mayor } \\
\text { distribución de Energía. (1pt) }\end{array}$ & 4 \\
\hline & $\begin{array}{l}\text { Realiza un bosquejo del grafico de Distribución de } \\
\text { Energía vs Longitud de Onda (1pt) }\end{array}$ & 1 \\
\hline & Intenta graficar (1pt) & 0.5 \\
\hline
\end{tabular}

\section{Cuál/es de las siguientes afirmaciones es/son correctas: (1 Punto)}

I) La ley de Rayleigh-Jeans coincide con la distribución de Planck a altas frecuencias.

II) Para Rayleigh-Jeans la contribución de los osciladores de alta frecuencia conduciría a la catástrofe ultravioleta.
a) Solo I
b) Solo II
c) I y II 